\title{
A novel non-Fermi-liquid state in the iron-pnictide FeCrAs
}

\author{
W. $\mathrm{Wu}^{1}$, A. McCollam ${ }^{1}$, I. Swainson ${ }^{2}$, P.M.C. Rourke ${ }^{1}$, D.G. Rancourt ${ }^{3}$ and S.R. Julian ${ }^{1}$ \\ 1 Department of Physics, University of Toronto, 60 St. George Street, Toronto, Canada M5S 1A7 \\ 2 Canadian Neutron Beam Centre, National Research Council Canada, Building 459, Chalk River Laboratories, Chalk \\ River, Ontario, Canada KOJ IJO \\ 3 Department of Physics, University of Ottawa, Ottawa, Ontario, Canada K1N 6N5
}

\begin{abstract}
PACS 71.10.Hf - Non-Fermi-liquid ground states, electron phase diagrams and phase tansitions in model systems

PACS 72.15.Qm - Scattering mechanisms and Kondo effect

PACS 72.80.Ga - Transition-metal compounds
\end{abstract}

\begin{abstract}
We report transport and thermodynamic properties of stoichiometric single crystals of the hexagonal iron-pnictide FeCrAs. The in-plane resistivity shows an unusual "non-metallic" dependence on temperature $T$, rising continuously with decreasing $T$ from $\sim 800 \mathrm{~K}$ to below 100 $\mathrm{mK}$. The $c$-axis resistivity is similar, except for a sharp drop upon entry into an antiferromagnetic state at $T_{N} \sim 125 \mathrm{~K}$. Below $10 \mathrm{~K}$ the resistivity follows a non-Fermi-liquid power law, $\rho(T)=$ $\rho_{0}-A T^{x}$ with $x<1$, while the specific heat shows Fermi liquid behaviour with a large Sommerfeld coefficient, $\gamma \sim 30 \mathrm{~mJ} / \mathrm{mol} \mathrm{K}^{2}$. The high temperature properties are reminiscent of those of the parent compounds of the new layered iron-pnictide superconductors, however the $T \rightarrow 0 \mathrm{~K}$ properties suggest a new class of non-Fermi liquid.
\end{abstract}

Introduction. - Metallic states that violate Landau's Fermi liquid paradigm appear in many strongly cor' related electron systems such as doped cuprates, quantum critical metals, and disordered Kondo lattices [1-3]. The new layered iron-pnictide high temperature superconductors also show non-Fermi-liquid behaviour, most notably in the undoped parent compounds which have a high, roughly constant resistivity at temperatures above a magnetic spin-density-wave transition that typically occurs around $T_{S D W} \sim 150 \mathrm{~K}$ (see, for example, [4-7]). For $T<T_{S D W}$, however, this incoherent charge transport gives way to a rapidly falling (i.e. "metallic") resistivity.

The basic structural units of the new iron-pnictide superconductors are layers in which iron atoms are tetrahedrally coordinated by arsenic. Some theoretical approaches to the incoherent $T>T_{S D W}$ state in these systems suggest that the physics is different from that of the cuprates, not least because this tetrahedral coordination produces only a small crystal-field splitting of the Fe $3 d-$ shell. Sawatzky et al. [8] argue that the electronic structure is dominated by narrow iron $3 d$ bands coupled to a highly polarizable arsenic environment. Dynamical mean field theory (DMFT) treatments [9-11] find an incoherent metallic state that arises from strong on-site inter-orbital interactions in the $3 d$-shell that perhaps puts some [9] or all [10] of the weakly crystal field split $3 d$-orbitals close to a Mott transition. The local physics of this so-called "bad semiconducting" state may represent a qualitatively new kind of incoherent metal.

In this paper we present magnetic, thermodynamic and transport properties of a ternary iron-arsenide, FeCrAs, whose behaviour shows intriguing similarities to the parent compounds of the layered iron-pnictide superconductors, including an antiferromagnetic transition in a similar temperature range $\left(T_{N} \sim 125 \mathrm{~K}\right)$. However there are key differences: firstly, the crystal structure is hexagonal as opposed to tetragonal; secondly, although the iron atoms are tetrahedrally coordinated by arsenic, FeCrAs is threedimensional (it does not have insulating layers); thirdly, as shown below, in FeCrAs the magnetism for $T<T_{N}$ resides primarily on the $\mathrm{Cr}$ sites, whereas in the layered iron-pnictides the magnetism is on the iron sites; and finally, the behaviour of the electrical resistivity of $\mathrm{FeCrAs}$ is much more extreme in that it is "non-metallic" over a huge temperature range - the in-plane resistivity rises with decreasing temperature from $\sim 800 \mathrm{~K}$ to below 100 $\mathrm{mK}$ without any sign of saturation or a gap at low temperature. Moreover, there is a profound and novel incompat- 
ibility at low temperature between the resistivity, which is non-Fermi-liquid with a sub-linear dependence on temperature, and the specific heat, which has a Fermi-liquid linear-in- $T$ dependence as $T \rightarrow 0 \mathrm{~K}$.

Experiment. - FeCrAs is a member of a large family of ternary $3 d$ transition metal monopnictides [12], many of which have a tetragonal crystal structure and order antiferromagnetically above room temperature. FeCrAs, however, has the hexagonal $\mathrm{Fe}_{2} \mathrm{P}$ structure, and generally members of this family with this structure order magnetically below room temperature, with ferromagnetic or complex antiferromagnetic order (see e.g. [13]).

Single crystals of FeCrAs were grown by melting stoichiometric quantities of high purity $\mathrm{Fe}, \mathrm{Cr}$ and As following the recipe given in [14]. Several batches of crystals were grown and annealed at $900^{\circ} \mathrm{C}$ in vacuum for five to ten days. Some batches were characterized by x-ray powder diffraction, which showed that the samples crystallized in the correct structure, with space group $\mathrm{P} \overline{6} 2 m$, and no detectable trace impurity phases. The crystals are shiny and metallic looking, but brittle. Material from one batch was examined by powder neutron diffraction at temperatures down to $2.8 \mathrm{~K}$. In addition to finding magnetic order at low temperature (discussed below) this showed that there is a high degree of site-order on the $\mathrm{Fe}$ and $\mathrm{Cr}$ sublattices. All measurements were performed on single crystals except for the x-ray and neutron diffraction.

Transport measurements used a conventional four terminal technique, either in a dilution refrigerator with a superconducting magnet for measurements below room temperature, or in a tube furnace under vacuum for high temperatures. In the latter measurements, the maximum temperature was limited by evaporation of the gold leads. Magnetic susceptibility measurements were done in a Quantum Design MPMS system, while specific heat measurements were done in a Quantum Design PPMS system.

Results. - The main panel in Fig. 1 shows the temperature dependence of resistivity. The absolute resistivity $\rho$ along the $a$ and $c$ axes ranges from $\sim 200$ to $\sim 500$ $\mu \Omega \mathrm{cm}$, which at high temperature at least is typical for a strongly correlated metal. There is only weak anisotropy in the resistivity, reflecting the absence of insulating layers in this material. The $a$-axis resistivity $\rho_{a}(T)$ shows a remarkable non-metallic behaviour, growing with decreasing temperature from $\sim 800 \mathrm{~K}$ down to at least $80 \mathrm{mK}$. An expanded plot of the $a$-axis resistivity at low temperature is shown in the Fig. 1 inset, where it can be seen that there is no sign of saturation or a gap that would give rise to exponentially increasing resistivity as $T$ falls. Instead, $\rho_{a}(T)$ has an exotic non-metallic and sub-linear in $T$ behaviour as $T \rightarrow 0 \mathrm{~K}: \rho_{a}(T)=\rho_{a, 0}-A T^{0.60 \pm 0.05}$.

The $c$-axis resistivity shows similar behaviour except that there is a very pronounced maximum, or cusp, at $\sim 125 \mathrm{~K}$. Examined closely, the in-plane resistivity also shows a slope change at approximately the same temper-

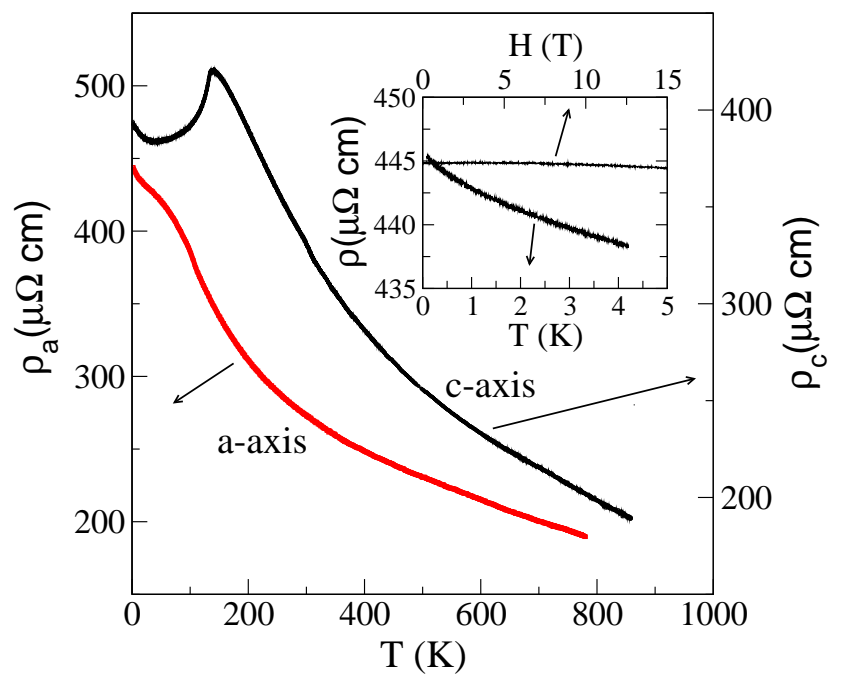

Fig. 1: Resistivity vs. temperature for $a$-axis (red curve) and $c$-axis (black curve). The inset shows the $a$-axis resistivity between $80 \mathrm{mK}$ and $4 \mathrm{~K}$ (bottom and left axes), demonstrating that it does not saturate or show a gap-like structure down to the lowest temperature measured, together with the resistivity vs. field at $170 \mathrm{mK}$ (upper and left axes), showing that the magnetoresistance is extremely weak .

ature. Below $10 \mathrm{~K} \rho_{c}(T)=\rho_{c, 0}-A T^{0.70 \pm 0.05}$, which is again sub-linear in $T$. At high temperatures, we followed the negative $d \rho / d T$ to nearly $900 \mathrm{~K}$ without finding any sign of saturation. Both the temperature at which the cusp appears and the magnitude of the drop in $\rho_{c}$ below this cusp have large sample to sample variations, however, the low temperature power law, and the resistivity above $\sim 150 \mathrm{~K}$, are sample independent.

The inset of Fig. 1 shows that the field dependence of $\rho$ is very weak, decreasing by $\sim 0.08 \%$ from 0 to 15 Tesla at $170 \mathrm{mK}$. We have also measured the Hall effect both at room temperature and between $80-300 \mathrm{mK}$, but find that the Hall signal is too small to be resolved. Based on the resolution of our devices, the low-temperature and room-temperature Hall coefficients are estimated to be less than $4.5 \times 10^{-3} \mathrm{~cm}^{3} / \mathrm{C}$ and $3.2 \times 10^{-3} \mathrm{~cm}^{3} / \mathrm{C}$, respectively. This suggests that $\mathrm{FeCrAs}$ is a compensated metal with a scattering rate that is uniform over the Fermi surface.

Fig. 2 shows the temperature dependence of the magnetic susceptibility $\chi$ for fields along $a$ - and $c$-axis, under both field cooled (FC) and zero field cooled (ZFC) conditions. For both orientations, a clear peak is observed at $125 \mathrm{~K}$, below which $\chi_{a}$ and $\chi_{c}$ deviate from one another. In addition, at lower temperatures a weak difference appears between the FC and ZFC susceptibilities, which is indicative of magnetic freezing as is observed in spin-glasses. In Fig. 2 this magnetic freezing sets in below $7 \mathrm{~K}$ and $15 \mathrm{~K}$ for $H \| c$ and $H \| a$ respectively; however, we have found that this behaviour is very sample dependent, starting as high as $\sim 45 \mathrm{~K}$ in some crystals. The 


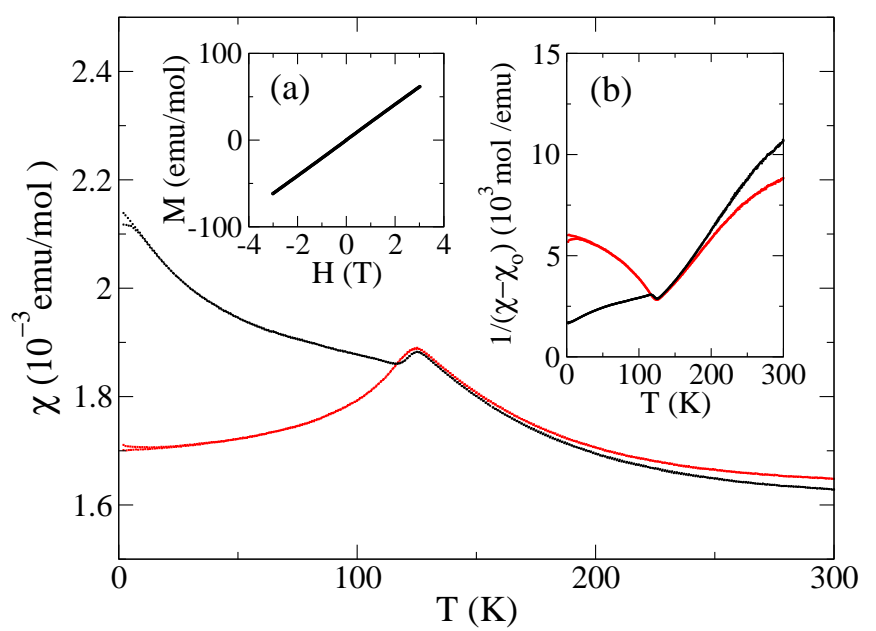

Fig. 2: Temperature dependence of the susceptibility $\chi$ for fields along $a$-axis (red curve) and $c$-axis (black curve). A small difference between zero-field-cooled and field-cooled conditions is visible at the lowest temperatures. Inset: (a) the temperature dependence of $1 /\left(\chi-\chi_{0}\right)$ showing an absence of CurieWeiss behaviour; (b) a hysteresis plot of the field dependence of the magnetization at $2 \mathrm{~K}$.

$\rho(T), C(T)$ and $\chi(T)$ data shown in this paper come from our "best" samples, that is, those with the lowest freezing temperature. However, we emphasize that, aside from the size of the drop in $\rho_{c}(T)$ below $125 \mathrm{~K}$ (which is largest in the "best" samples), none of these quantities showed qualitatively different behaviour between samples.

For all samples, no magnetic hysteresis was observed down to $2 \mathrm{~K}$ : the inset of Fig. 2 shows an example of the field dependence of the magnetization at $2 \mathrm{~K}$.

The overall change of the susceptibility is small in the whole temperature range observed, that is, $\chi(T)$ is quite Pauli-like and does not show Curie-Weiss temperature dependence (see the inset of Fig. 2) even after a temperature independent term $\chi_{0}$ has been subtracted to make $1 /(\chi$ $\left.\chi_{0}\right)$ as linear as possible.

Our powder neutron diffraction measurements found magnetic peaks below $\sim 100 \mathrm{~K}$, demonstrating that the $125 \mathrm{~K}$ features in $\chi(\mathrm{T})$ and $\rho_{c}(T)$ arise from magnetic ordering. The temperature dependence of $\chi$ and the hysteresis measurement at $2 \mathrm{~K}$ indicate that this ordering is antiferromagnetic. Indeed, the magnetic peaks in the neutron diffraction were indexed to a propagation vector of $\boldsymbol{k}=\left(\frac{1}{3}, \frac{1}{3}, 0\right)$ corresponding to a commensurate spindensity wave that triples each in-plane unit cell dimension. Fitting the neutron spectra gives a moment on the $\mathrm{Cr}$ sites that varies between a maximum of of 2.2 and a minimum of $0.6 \mu_{B}$. The moment on the iron sites is much weaker, and indeed within the noise it is consistent with an earlier Mössbauer spectroscopy measurement [15] which found very weak magnetic order on the iron sites at $4.2 \mathrm{~K}$, with the magnetic moment estimated to be 0.1 $\pm 0.03 \mu_{B}$. A full treatment of the magnetic structure and symmetry analysis will be published separately.

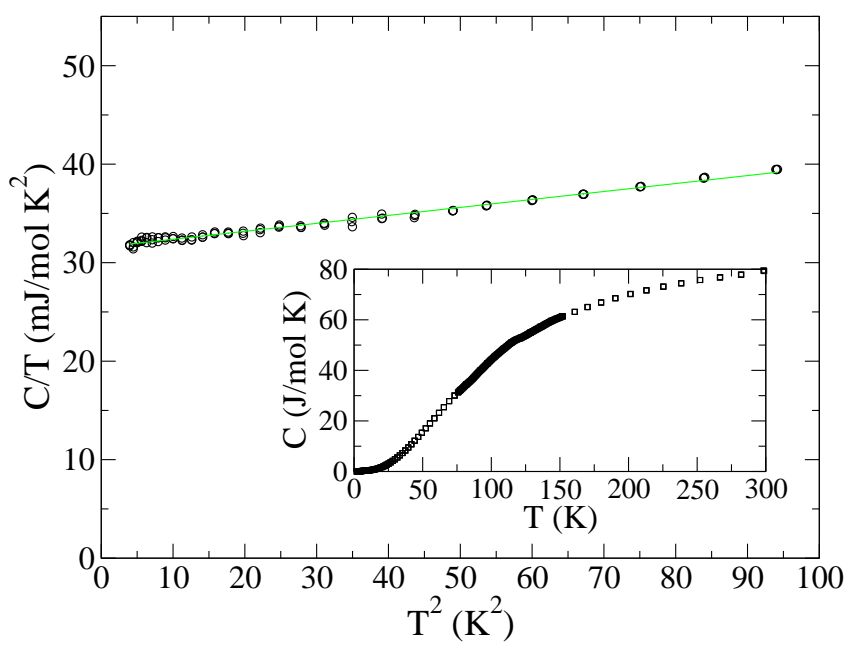

Fig. 3: $\mathrm{C} / \mathrm{T}$ vs $T^{2}$ at low temperatures (circles). The green line is a linear fit. The inset shows $C(T)$ from room temperature to $2 \mathrm{~K}$. The Néel transition produces a weak anomaly at $125 \mathrm{~K}$.

In striking contrast to the resistivity, the specific heat, shown in Fig. 3, exhibits classic Fermi liquid behaviour at low temperatures: $C(T) / T$ depends linearly on $T^{2}$. The Sommerfeld coefficient, $\gamma=C_{e l} / T$, extracted from the data in Fig. 3 gives a value of $31.6 \mathrm{~mJ} / \mathrm{mol} \mathrm{K}^{2}$, which is quite high for a $d$-electron system. We find that $\gamma$ varies between samples by up to $20 \%$, however the linear relationship of $C(T) / T$ vs $T^{2}$ holds in all samples down to at least $1 \mathrm{~K}$. At high temperatures, the specific heat shows a weak anomaly near the Néel temperature (see inset of Fig. (3).

To compare the enhancements of the specific heat coefficient and the spin susceptibility, the Wilson ratio, $R_{W}=K \chi / \gamma$, is commonly applied, where $K$ is a scale factor which gives a dimensionless value $R_{W}=1$ for a free electron gas. For a Kondo system, a value of $R_{W}=2$ is expected. In FeCrAs, however, we find $R_{W} \sim 5$ from the $c$-axis susceptibility and $R_{W} \sim 4$ from the $a$-axis susceptibility.

Discussion. - The unusual features of our results are as follows: 1) both the in-plane and $c$-axis resistivities show strong non-metallic behaviour with negative $\mathrm{d} \rho / \mathrm{d} T$ to beyond $\sim 800 \mathrm{~K}$, the highest temperatures we measured; 2) at low temperatures, both the in-plane and $c$-axis resistivities have strongly non-metallic, non-Fermi-liquid behaviour, while the specific heat is Fermi-liquid like; 3) the linear coefficient of specific heat $\gamma$ is unusually large for a $d$-electron material; 4) there is a large, roughly temperature independent (i.e. Pauli-like) susceptibility, giving a Wilson ratio of between 4 and 5; and 5) the ordered moment on the iron sites is unusually small for an iron compound.

Some of these features are common to the parent compounds of the new iron-pnictide superconductors, notably the large value of $\gamma$, the high resistivity above the mag- 
netic ordering transition, the weakness of magnetism on the iron sites, the Pauli-like susceptibility and the high Wilson ratio (see e.g. [4-7]). Even the magnetic ordering transition temperature is similar, however the magnetism here is of a very different origin: FeCrAs adopts a $\left(\frac{1}{3}, \frac{1}{3}, 0\right)$ in-plane antiferromagnetic structure but this ordering is clearly driven by the chromium sublattice, while in the layered pnictides the SDW-magnetic order is driven by the Fe sites. This may explain why the incoherent transport in the layered iron-pnictides disappears below $T_{S D W}$, while in $\mathrm{FeCrAs}$ it continues to $T \rightarrow 0 \mathrm{~K}$. (It should be noted that in polycrystalline LaFeOAs the resisitivity at low temperature does show an upturn as $T \rightarrow 0 \mathrm{~K}$, which may be intrinsic or a grain boundary effect [4].)

The similarities suggest that there may be physics that is common to the incoherent states of both FeCrAs and the layered iron-pnictides. For example, the fall in $\rho_{c}(T)$ just below $T_{N} \sim 125 \mathrm{~K}$ is similar to that for $T<T_{S D W}$ in the layered materials, and may be a signature of a spinfluctuation contribution to the resistivity. Dai et al. [16] have suggested that the incoherence of the layered ironpnictides arises from spin-fluctuation scattering enhanced by frustrated exchange interactions. Similar arguments may apply in FeCrAs, and indeed the crystal structure of $\mathrm{FeCrAs}$ is more amenable to frustration than that of the layered iron-pnictides.

The $\mathrm{Fe}_{2} \mathrm{P}$ crystal structure can be viewed in a number

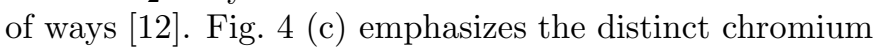
(blue atoms) and iron (green atoms) sublattices. The $\mathrm{Cr}$ sublattice, which carries the bulk of the magnetic order, can be viewed as a distorted Kagome lattice, while the iron atoms form "trimers", the green triangles of Fig. 4, that lie on a triangular lattice. These planes of iron trimers and the chromium Kagome planes are alternately stacked along the $c$-axis, and the triangular network of trimers in particular offers interesting possibilities for frustration. We note that the small ordered Fe magnetic moment, the very weak dependence of $\rho(\mathrm{T}), \chi(\mathrm{T})$ and $C(T) / T$ on magnetic field, and signatures of magnetic freezing in these crystallographically well-ordered crystals, are all typical of magnetically frustrated systems $[17,18]$.

However, the antiferromagnetic order is a major complication in this scenario, as is the absence of Curie-Weiss susceptibility. An even more serious difficulty is the energy scale: the related tetragonal materials $\mathrm{Fe}_{2} \mathrm{As}$ and $\mathrm{Cr}_{2} \mathrm{As}$ order antiferromagnetically at $350 \mathrm{~K}$ [19] and $393 \mathrm{~K}$ [20]. If we take this as the typical scale of the exchange energy $J$ in these systems, it is then difficult to see how the nonmetallic resistivity could persist to temperatures that are twice as high. In most spin fluctuation systems the resistivity is a rising function of temperature, and it saturates when $T$ is larger than $J$. Finally, we note that the weak magnetism on the iron sites is probably due to electronic structure effects, not magnetic frustration. LMTO calculations by Ishida et al. [21] consistently find for the $\mathrm{Fe}_{2} \mathrm{P}$ structure that the tetrahedrally coordinated site (the $\mathrm{Fe}$ site in FeCrAs) has a small moment, while the "pyrami-
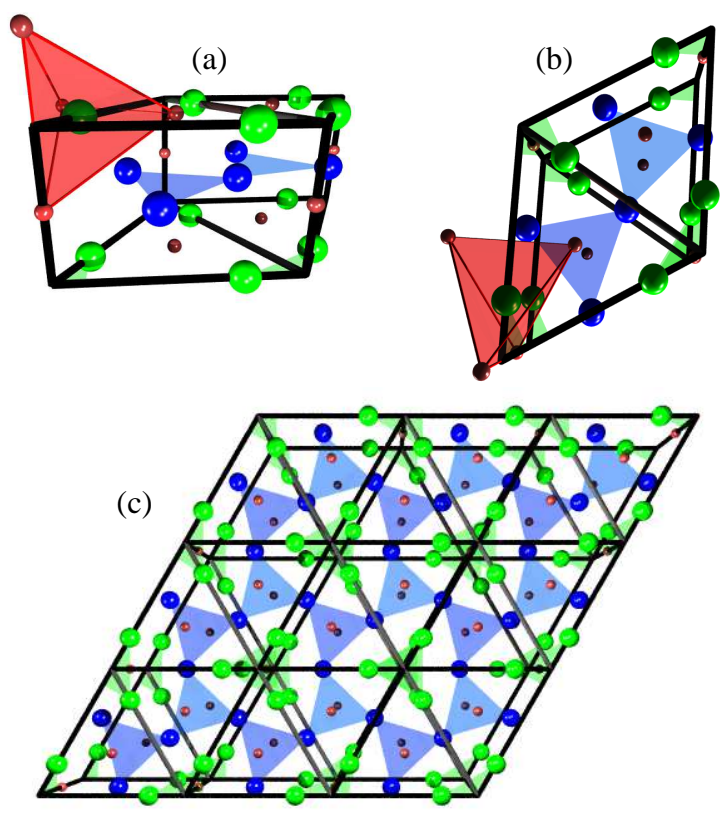

Fig. 4: Crystal structure of FeCrAs. Arsenic atoms are shown as small red balls, $\mathrm{Cr}$ atoms are blue and $\mathrm{Fe}$ atoms are green. (a) and (b): the primitive unit cell from the side and the top respectively. The red tetrahedron shows the tetrahedral coordination of iron by arsenic. (c): looking down the $c$-axis at a three by three slice. The network of blue triangles illustrates the distorted Kagome lattice on which the Cr atoms sit, while the green triangles illustrate the triangular lattice of $\mathrm{Fe}$ "trimers".

dal" site, coordinated by five As atoms (the Cr site in $\mathrm{FeCrAs}$ ), has a large moment. Indeed for FeCrAs they find that the density of states of the $3 d$-bands of $\mathrm{Fe}$ is below the Stoner criterion, and although they found that a ferromagnetic ground state is energetically favoured, the antiferromagnetic state that they used for comparison was quite different from the one that we have found.

A very different explanation of the high temperature resistivity might be the local physics arising on the tetrahedrally coordinated Fe sites [8-11]. The bad semiconductor/bad metal picture of the incoherent metallic state of the layered iron-pnictides that arises in DMFT calculations is appealing because the energy scale is not the intersite exchange coupling $J$. Rather it is the on-site interorbital Coulomb interaction, estimated to be on the order of a few eV [8]. We therefore believe that FeCrAs may be an interesting system for exploring this new physics. We emphasize, however, that the strong temperature dependence of the non-metallic resistivity of FeCrAs is different from the roughly temperature independent resistivity seen at high temperatures in the parent compounds of the ironpnictide superconductors.

Whether the DMFT picture explains the low temperature limit is not clear. Laad et al. [11], in attempting to explain optical conductivity, find a scattering rate that is sub-linear in $\omega$, which may translate into a resistivity 
that is sub-linear in $T$. However, the most striking feature of our low temperature results - the incompatibility of the non-metallic and non-Fermi-liquid resistivity with the classic Fermi-liquid specific heat - has not been discussed within either the spin-fluctuation or the DMFT pictures.

There are a number of materials that show strong nonmetallic resistivity over a large temperature range, terminating at low temperature in a non-Fermi-liquid state. Underdoped cuprates are an obvious example [22], however in that case the $T \rightarrow 0 \mathrm{~K}$ limit of $\rho(T)$ has a logarithmic divergence, not power-law behaviour.

Some disordered heavy fermion systems $[3,23]$ have non-metallic resistivity over a large temperature range, with low temperature linear-in- $T$ behaviour, however in all of these cases the specific heat also shows strong nonFermi-liquid behaviour as $T \rightarrow 0 \mathrm{~K}$, typically $C(T) / T \sim$ $-\ln (T)$. For example, in $\mathrm{CeRh}_{2} \mathrm{Ga}, C(T) / T$ is logarithmic in $T$ and rises from $\sim 150 \mathrm{~mJ} / \mathrm{mol} \mathrm{K}^{2}$ to $\sim 480 \mathrm{~mJ} / \mathrm{mol} \mathrm{K}^{2}$ between $6 \mathrm{~K}$ and $2 \mathrm{~K}$ [24]. In contrast, the specific heat of $\mathrm{FeCrAs}$ is only weakly temperature dependent at low temperature and it is linear-in- $T$ as $T \rightarrow 0 \mathrm{~K}$. Moreover, the disordered Kondo systems show dramatic rises in $\chi(T)$ over the whole temperature range, typically by a factor of around 10 between room temperature and $1 \mathrm{~K}$, with increasing slope as $\mathrm{T} \rightarrow 0 \mathrm{~K}$. The Pauli-like $\chi(T)$ of FeCrAs is completely different. We note additionally that the disordered Kondo systems have a large magnetoresistance at low temperature, while FeCrAs does not.

It should be noted that there is one intriguing exception among the heavy fermion materials to the divergent $C(T) / T$ accompanying a non-metallic non-Fermi-liquid resistivty, and that is another arsenide, $\mathrm{CeCuAs}_{2}$, in which $C(T) / T$ exhibits a fall, instead of logarithmic increase, below $2 \mathrm{~K}[25]$. However in this material too the susceptibility rises very strongly at low temperature, unlike FeCrAs. The under-screened Kondo effect has been suggested as an explanation of the non-metallic non-Fermi-liquid resistivity in $\mathrm{CeCuAs}_{2}$ [26], but given the apparent quenching of iron local moments by tetrahedral coordination with As $[8,21]$, this scenario seems unlikely in FeCrAs.

Finally, disorder-induced localization effects, which have been invoked to explain non-metallic non-Fermiliquid behaviour in the moderately site-disordered $U$ heavy fermion compounds $\mathrm{URh}_{2} \mathrm{Ge}_{2}[27]$ and $\mathrm{UCu}_{4} \mathrm{~Pb}[28]$ in the range of $\sim 1 \mathrm{~K}$ to $300 \mathrm{~K}$, seem to be ruled out because those materials exhibit quite a large magnetoresistance, clear signals in the Hall effect, and non-Fermi-liquid behaviours in heat capacity and magnetic susceptibility, while FeCrAs has none of these properties.

Thus we believe that the behaviour of $\mathrm{FeCrAs}$ is qualitatively different from any previous observations of nonmetallic, non-Fermi-liquid behaviour in the $T \rightarrow 0 \mathrm{~K}$ limit.

It is natural to hypothesize that the linear lowtemperature specific heat arises from fermionic excitations that are distinct from the non-metallic non-Fermi-liquid charge carriers. Such "neutral" fermions arise in some theories of fractionalization in insulating, frustrated spin liquids [29-31], and may recently have been observed experimentally [32]. Fractionalization has also been introduced to describe antiferromagnetic quantum criticality in some heavy fermion compounds [33,34]. A key ingredient of most fractionalization scenarios is a geometrically frustrated spin system (see e.g. [35]). We have noted some difficulties with this picture in $\mathrm{FeCrAs}$, but it may be relevant that the DMFT studies of the layered ironpnictides $[9,11]$ find "orbitally selective" effects that can be tuned by varying the on-site interactions. Although it is not believed that any of the $3 d$-orbitals of Fe in the layered iron-pnictides are Mott localized, the local environment of the iron sites in FeCrAs may be sufficiently different from the layered systems that some of the $3 d$-orbitals do localize, producing local degrees of freedom that do not contribute to charge transport. It is far from clear, however, if these degrees of freedom would be fermionic at low temperatures.

Conclusion. - In summary, FeCrAs is an unusual $d$ electron system in which non-Fermi-liquid behaviour has been observed. Its resistivity shows non-Fermi-liquid behaviour obeying $\rho(T)=\rho_{0}-A T^{x}$ at low temperatures with $x=0.60 \pm 0.05$ for $\rho_{a}$ and $x=0.70 \pm 0.05$ for $\rho_{c}$, while at high temperatures $\rho$ decreases with increasing temperature up to at least $800 \mathrm{~K}$. The low temperature specific heat, on the other hand, exhibits typical Fermiliquid behaviour with a linear temperature dependence. This low temperature state is very robust in the sense that it exhibits very weak dependence on field, and the same behaviour is observed in all the samples we have measured. The high temperature behaviour may be an extreme example of the "bad semiconductor" state found by DMFT calculations for the layered iron-pnictides, and although the low temperature state may have the same origin, it deserves further study as it has some features expected of a fractionalized electron system which have not, to our knowlege, been observed before.

$$
* * *
$$

We are grateful to H.Y. Kee, J. Hopkinson, A.P. Mackenzie and Z. Tesanovic for valuable discussions and to Y.J. Kim and F. Wang for assistance in our magnetic hysteresis and susceptibility measurements.

\section{REFERENCES}

[1] Hussey N. E., J. Phys. Cond. Mat., 20 (2008) 123201.

[2] von Lohneysen H., Rosch A., Vojta M. and Wolfle P., Rev. Mod. Phys., 79 (2007) 1015.

[3] Stewart G. R., Rev. Mod. Phys., 73 (2001) 797.

[4] Kamihara Y., Watanabe T., Hirano M. and Hosono H., J. Am. Chem. Soc., 130 (2008) 3296.

[5] Ren Z. A., Lu W., Yang J., Yi W., Shen X. L., Li Z., Che G. C., Dong X. L., Sun L. L., Zhou F. and ZhaO Z. X., Chinese Physics Letters, 25 (2008) 2215. 
[6] Rotter M., Tegel M. and Johrendt D., Phys. Rev. Lett. , 101 (2008) 107006.

[7] Sasmal K., Lv B., Lorenz B., Guloy A. M., Chen F., Xue Y.-Y. and Chu C.-W., Phys. Rev. Lett. , 101 (2008) 107007.

[8] Sawatzky G. A., Elfimov I. S., van den Brink J. and ZAANEN J., arXiv:0808.1390.

[9] Shorikov A. O., Korotin M. A., Streltsov S. V., Skornyakov S. L., Korotin D. M. and Anisimov V. I., arXiv:0804.3283.

[10] Haule K., Shim J. H. and Kotliar G., Phys. Rev. Lett. , 100 (2008) 226402.

[11] Laad M. S., Craco L., Leoni S. and Rosner H., arXiv:0810.1607.

[12] Fruchart R., Ann. Chim. Fr. , 7 (1982) 563.

[13] Bacmann M., Fruchart D., Koumina A. and Wolfers P., Materials Science Forum , 443-4 (2004) 379 .

[14] Katsuraki H. and Achiwa N., J. Phys. Soc. Japan., 21 (1966) 2238.

[15] Rancourt D. G., Hyperfine field fluctuations in the Mössbauer spectrum of magnetic materials: Application to small particles and to the bulk antiferromagnet $\mathrm{Fe}_{(2-\mathrm{x})} \mathrm{Cr}_{\mathrm{x}}$ As. Ph.D. thesis University of Toronto (1984).

[16] Dai J., Si Q., Zhu J.-X. and Abrahams E., arXiv:0808.0305.

[17] Nakatsuji S., Nambu Y., Tonomura H., Sakai O., Jonas S., Broholm C., Tsunetsugu H., Qiu Y. and Maeno Y., Science, 309 (2005) 1697.

[18] Saunders T. E. and Chalker J. T., Phys. Rev. Lett. , 98 (2007) 157201.

[19] Katsuraki H., J. Phys. Soc. Japan. , 19 (1964) 1988.

[20] Yuzuri M., J. Phys. Soc. Japan. , 15 (1960) 2007.

[21] Ishida S., Takiguchi T., Fujil S. and Asano S., Physica $B, \mathbf{2 1 7}$ (1996) 87.

[22] Ando Y., Boebinger G. S., Passner A., Kimura T. and Kishio K., Phys. Rev. Lett. , 75 (1995) 4662.

[23] Maple M. B., de Andrade M. C., Herrmann J., Dalichaouch Y., Gajewski D. A., Seaman C. L., Chau R., Movshovich R., Aronson M. C. and OsBOrn R., J. Low Temp. Phys., 99 (1995) 223.

[24] Chen D. F., Sakamoto I., Ohara S., Takami T., Ikuta H. and Mizutani U., Phys. Rev. B , 69 (2004) 014420.

[25] Sengupta K., Sampathkumaran E. V., Nakano T., Hedo M., Abliz M., Fujiwara N., Uwatoko Y., Rayaprol S., Shigetoh K., Takabatake T., Doert T. and Jemetio J. P. F., Phys. Rev. B , 70 (2004) 064406.

[26] Florens S., Phys. Rev. B , 70 (2004) 165112.

[27] Sullow S., Maksimov I., Оtop A., Litterst F. J., Perucchi A., Degiorgi L. and Mydosh J. A., Phys. Rev. Lett. , 93 (2004) 266602.

[28] Отор A., Sullow S., Maple M. B., Weber A., Scheidt E. W., Gortenmulder T. J. and Mydosh J. A., Phys. Rev. B, 72 (2005) 024457.

[29] Lee P. A., Nagaosa N. and Wen X. G., Rev. Mod. Phys. , 78 (2006) 17.

[30] Affleck I. and Marston J. B., Phys. Rev. B , 37 (1988) 3774.

[31] Baskaran G. and Anderson P. W., Phys. Rev. B , 37 (1988) 580.
[32] Kagawa F., Miyagawa K. and Kanoda K., Nature, 436 (2005) 534.

[33] Pepin C., Phys. Rev. Lett. , 94 (2005) 066402.

[34] Senthil T., Sachdev S. and Vojta M., Phys. Rev. Lett. , 90 (2003) 216403.

[35] Moessner R. and Sondhi S. L., Phys. Rev. Lett. , 86 (2001) 1881. 